PROCEEDINGS OF THE

AMERICAN MATHEMATICAL SOCIETY

Volume 134, Number 2, Pages 427-434

S 0002-9939(05)08196-7

Article electronically published on September 20, 2005

\title{
ON THE RIGIDITY OF MAGNETIC SYSTEMS WITH THE SAME MAGNETIC GEODESICS
}

\author{
KEITH BURNS AND VLADIMIR S. MATVEEV
}

(Communicated by Michael Handel)

\begin{abstract}
We study the analogue for magnetic flows of the classical question of when two different metrics on the same manifold share geodesics, which are the same up to reparametrization.
\end{abstract}

\section{INTRODUCTION}

Let $M$ be a compact smooth manifold and let $\pi: T M \rightarrow M$ be the canonical projection from the tangent bundle to $M$. A magnetic structure on $M$ is a pair $(g, \Omega)$, where $g$ is a Riemannian metric and $\Omega$ is a closed 2-form on $M$. Let $\omega_{g}$ be the symplectic form on $T M$ obtained by pulling back the canonical symplectic form on $T^{*} M$ via the Riemannian metric $g$. The magnetic flow for $(g, \Omega)$ is the Hamiltonian flow $\varphi_{t}$ on $T M$ determined by the symplectic form

$$
\omega_{m a g}=\omega_{g}+\pi^{*} \Omega
$$

and the function

$$
E(v)=\frac{1}{2} g(v, v)
$$

The magnetic flow models the motion of a charged particle under the effect of a magnetic field, whose Lorentz force $Y: T M \rightarrow T M$ is the bundle map defined by

$$
\Omega_{x}(u, v)=g_{x}\left(Y_{x}(u), v\right),
$$

for all $x \in M$ and all $u$ and $v$ in $T_{x} M$. The orbits of the magnetic flow have the form $t \mapsto \dot{\gamma}(t)$, where $\gamma$ is a curve in $M$ such that

$$
\frac{D \dot{\gamma}}{d t}=Y_{\gamma}(\dot{\gamma})
$$

where $D / d t$ denotes the covariant derivative of $g$ along $\gamma$. The magnetic flow of the pair $(g, 0)$ is the geodesic flow of the Riemannian metric $g$. A curve $\gamma$ that satisfies equation (10) will be called a magnetic geodesic. The magnetic flow shares with the usual Riemannian geodesic flow the property that the level sets of the energy function $E$ are preserved. A magnetic geodesic is the path followed by a particle with unit mass and charge under the effect of the magnetic field. If we

Received by the editors September 5, 2004.

2000 Mathematics Subject Classification. Primary 37Dxx, 37D40, 37Jxx, 53B10.

The first author was supported by NSF grants DMS-9803346 and DMS-0100416.

The second author was supported by DFG-programm 1154 (Global Differential Geometry) and Ministerium für Wissenschaft, Forschung und Kunst Baden-Württemberg (Eliteförderprogramm Postdocs 2003).

(C)2005 American Mathematical Society
Reverts to public domain 28 years from publication 427 
reparametrize a magnetic geodesic corresponding to energy $h$ so that it has unit speed, then we obtain the path followed by a unit mass particle whose charge is inversely proportional to $\sqrt{h}$. It is often convenient to think of the Riemannian geodesics as magnetic geodesics corresponding to infinite energy.

Many questions for geodesic flows have natural counterparts for magnetic flows which have been studied recently. See e.g. [2, 3, 4, 45, 8, 12, 13, 15, 14, 16, 20, 21,

A classical topic in the theory of geodesic flows is the question of when two different metrics $g$ and $\bar{g}$ on the same manifold can share the same geodesics, in the sense that every $\bar{g}$-geodesic is a reparametrization of a $g$-geodesic and conversely. Let us recall a few simple examples (which will have magnetic analogs).

First of all, the metrics $g$ and $\bar{g}=\alpha \cdot g$, where $\alpha>0$ is a constant, clearly have the same geodesics.

Another simple example is as follows. Let $\left(M_{1}, g_{1}\right)$ and $\left(M_{2}, g_{2}\right)$ be two Riemannian manifolds, and consider the product metrics $g_{1}+g_{2}$ and $\alpha_{1} \cdot g_{1}+\alpha_{2} \cdot g_{2}$ on $M_{1} \times M_{2}$, where $\alpha_{1}, \alpha_{2}$ are constants. These metrics are evidently affinely equivalent (i.e. they have the same Christoffel symbols), and therefore have the same geodesics.

The first nontrivial example appeared in Beltrami [1: the metric $g$ is the restriction of the Euclidean metric $d x^{2}+d y^{2}+d z^{2}$ to the sphere

$$
S^{2}=\left\{(x, y, z) \in R^{3}: x^{2}+y^{2}+z^{2}=1\right\} .
$$

The metric $\bar{g}$ is the pull-back $l^{*} g$, where the mapping $l: S^{2} \rightarrow S^{2}$ is given by $l: v \mapsto \frac{A(v)}{\|A(v)\|}$, where $A$ is an arbitrary linear nondegenerate transformation of $R^{3}$.

The metrics $g$ and $\bar{g}$ have the same (unparametrized) geodesics. Indeed, the geodesics of the metric $g$ are great circles (the intersections of planes that go through the origin with the sphere). The mapping $A$ is linear and therefore takes the planes to the planes. Since the normalization $w \mapsto \frac{w}{\|w\|}$ takes the planes to their intersections with the sphere, the mapping $l$ takes the great circles to the great circles. Thus, any geodesic of the metric $g$ is a reparametrized geodesic of $\bar{g}$. Evidently, if $A$ is not proportional to an orthogonal transformation, the metrics $g$ and $\bar{g}$ are not affinely equivalent.

This paper considers the analogous question for magnetic flows: when do two different magnetic systems on the same manifold share magnetic geodesics which are the same up to reparametrization?

Lichnerowicz and Aufenkamp considered a generalized version of this question in 6, 7]. They showed that the assumption that two magnetic systems have the same geodesic is equivalent to a complicated nonlinear system of partial differential equations.

The three examples of Riemannian metrics sharing the same geodesics described above have magnetic analogs.

The magnetic geodesics of $(g, \Omega)$ coincide up to reparametrization with those of the rescaled system $(\alpha \cdot g, \beta \cdot \Omega)$, for any constants $\alpha>0, \beta \neq 0$.

More generally the factors in a direct product of magnetic structures can be rescaled independently: if $\left(M_{1}, g_{1}, \Omega_{1}\right)$ and $\left(M_{2}, g_{2}, \Omega_{2}\right)$ are two manifolds with magnetic structures, any magnetic geodesic of the magnetic structure $\left(g_{1}+g_{2}\right.$, $\Omega_{1}+\Omega_{2}$ ) on the product $M_{1} \times M_{2}$ is a reparametrization of a magnetic geodesic of $\left(\alpha_{1} g_{1}+\alpha_{2} g_{2}, \beta_{1} \Omega_{1}+\beta_{2} \Omega_{2}\right)$, where $\alpha_{1}>0, \alpha_{2}>0, \beta_{1} \neq 0$ and $\beta_{2} \neq 0$ are constants. 
There is an analogue of Beltrami's example for magnetic systems. Consider the sphere $S^{2}$ and the magnetic system $(g, \Omega)$ on it, where $g$ is the round metric and $\Omega$ is the corresponding volume form. Consider the standard complex structure on the sphere, an arbitrary linear-fractional transformation $l: S^{2} \rightarrow S^{2}$ and the magnetic system $\left(l^{*} g, l^{*} \Omega\right)$. Because of the symmetries of the system, the magnetic geodesics of $(g, \Omega)$ are circles. The linear-fractional transformation $l$ takes circles to circles and therefore the magnetic system $(g, \Omega)$ has the same (unparametrized) geodesics as the magnetic system $\left(l^{*} g, l^{*} \Omega\right)$.

The first of the above examples has the property that curves which are magnetic geodesics with the same energy for one system are also magnetic geodesics with the same energy for the other system. In particular, the Riemannian geodesics of the first system are Riemannian geodesics of the second system up to reparametrization. In the second example, magnetic geodesics with the same energy for one system usually do not have the same energy in the other system, but it is still true that Riemannian geodesics of the first system are Riemannian geodesics of the second system up to reparametrization. The Beltrami example has neither of the properties.

Our main result is that the first example is essentially the only case in which two magnetic systems can share the same magnetic geodesics in such a way that an energy level of one system corresponds to an energy level of the other system.

Theorem 1.1. Let $(g, \Omega)$ and $(\bar{g}, \bar{\Omega})$ be two magnetic systems on the same connected manifold. Suppose that there are positive constants $h$ and $\bar{h}$ such that every magnetic geodesic for $(g, \Omega)$ with energy $h$ is a reparametrization of a magnetic geodesic for $(\bar{g}, \bar{\Omega})$ with energy $\bar{h}$. Then $(g, \Omega)$ and $(\bar{g}, \bar{\Omega})$ are rescalings of one another, or $\Omega=0=\bar{\Omega}$ and $g$ and $\bar{g}$ are metrics with the same geodesics.

We apply Theorem 1.1 to extend to magnetic flows a recent result of Matveev and Topalov [9, 10, 17.

Theorem 1.2. Assume the dimension of the manifold $M$ is at least two. Suppose that there is a magnetic geodesic for $(g, \Omega)$ whose tangent vectors are dense in their energy level. Then the only magnetic systems with the same magnetic geodesics as $(g, \Omega)$ are rescalings of $(g, \Omega)$.

The first author thanks the Universität Freiburg for their hospitality during the work on this paper.

\section{Proofs of the theorems}

2.1. Proof of Theorem 1.1, Let $M$ be the manifold in question. Without loss of generality we can assume that the dimension of the manifold is at least two. Let $E(v)=\frac{1}{2} g(v, v)$ and $\bar{E}(v)=\frac{1}{2} \bar{g}(v, v)$ be the energies associated to the systems and define

$$
S_{g, h}=\{v \in T M: E(v)=h\} \quad \text { and } \quad S_{\bar{g}, \bar{h}}=\{v \in T M: \bar{E}(v)=\bar{h}\} .
$$

For nonzero $v$, let

$$
c(v)=\frac{\sqrt{h}}{\sqrt{h}} \frac{|v|_{g}}{|v|_{\bar{g}}} .
$$

Lemma 2.1. Suppose $v \in S_{g, h}$ and $c v \in S_{\bar{g}, \bar{h}}$. Then $c= \pm c(v)$. 
Proof. Since $v \in S_{g, h}$ and $c v \in S_{\bar{g}, \bar{h}}$, we have $|v|_{g}=\sqrt{2 h}$ and $|c v|_{\bar{g}}=\sqrt{2 \bar{h}}$. Hence

$$
|c| \frac{|v|_{\bar{g}}}{|v|_{g}}=\frac{\sqrt{h}}{\sqrt{h}} .
$$

The lemma follows.

Define the map $\psi: S_{g, h} \rightarrow S_{\bar{g}, \bar{h}}$ by

$$
\psi(v)=c(v) v
$$

We can extend $\psi$ to a map of $T M$ to itself that is homogeneous of degree one, which we again denote by $\psi$. We will often write $\bar{v}$ for $\psi(v)$.

It is easy to see from the hypothesis of Theorem 1.1 that either $\psi$ maps orbits of the $(g, \Omega)$ structure in $S_{g, h}$ to orbits of the $(\bar{g}, \bar{\Omega})$ in $S_{\bar{g}, \bar{h}}$ or the map $v \mapsto-\psi(v)$ has this property. We shall assume that $\psi$ has this property. The other case can be reduced to the one we consider by multiplying $\bar{\Omega}$ by -1 .

Let $X_{m a g}$ and $X_{g e o}$ denote the generators of the magnetic flow for $(g, \Omega)$ and the geodesic flow for $g$, respectively. The corresponding objects for the $(\bar{g}, \bar{\Omega})$ structure will be denoted by $\bar{X}_{m a g}$ and $\bar{X}_{g e o}$. The Lorenz forces associated with the two magnetic structures will be denoted by $Y$ and $\bar{Y}$, respectively.

For a vector $v \in T_{p} M$ let $i_{v}: T_{p} M \oplus T_{p} M \rightarrow T_{v} T M$ be the standard linear isomorphism determined by the metric $g$, i.e. $i_{v}^{-1}(\xi)=(d \pi(\xi), K(\xi))$, where $d \pi$ is the differential of the projection $\pi: T M \rightarrow M$ and $K$ is the connector map defined by $K(\xi) \stackrel{\text { def }}{=} \frac{D V}{d t}(0)$, where $V(t)$ is a curve in $T M$ with $\dot{V}(0)=\xi$ and $\frac{D}{d t}$ denotes the covariant derivative along the curve $\pi(V)$.

Then

$$
X_{g e o}=i_{v}(v, 0) \quad \text { and } \quad X_{m a g}=i_{v}\left(v, Y_{\pi(v)}(v)\right) .
$$

In particular $d \pi \circ X_{g e o}$ and $d \pi \circ X_{m a g}$ are both the identity map. Analogous properties hold for $\bar{X}_{g e o}$ and $\bar{X}_{m a g}$.

Lemma 2.2. $\bar{X}_{\text {mag }}(\bar{v})=c(v) d \psi\left(X_{\text {mag }}(v)\right)$ for all $v \in S_{g, h}$.

Proof. Since $\psi$ carries orbits of the $(g, \Omega)$ magnetic flow in $S_{g, h}$ to orbits of the $(\bar{g}, \bar{\Omega})$ magnetic flow in $S_{\bar{g}, \bar{h}}$, there is a function $C: S_{g, h} \rightarrow \mathbf{R}$ such that

$$
\bar{X}_{m a g}(\bar{v})=C(v) d \psi\left(X_{m a g}(v)\right)
$$

for all $v \in S_{g, h}$. We now show that $C(v)=c(v)$ for all $v \in S_{g, h}$. Projecting from $T T M$ to $T M$ gives

$$
d \pi \circ \bar{X}_{\text {mag }}(\bar{v})=C(v) d \pi \circ d \psi\left(X_{\text {mag }}(v)\right) .
$$

Note that $d \pi \circ \psi=d \pi$, because $\psi$ maps fibres of the tangent bundle into themselves. Hence

$$
d \pi \circ \bar{X}_{m a g}(\bar{v})=\bar{v}=c(v) v
$$

and

$$
d \pi \circ d \psi \circ X_{m a g}(v)=d \pi \circ X_{m a g}(v)=v .
$$

We see from the last three equations that $C(v)=c(v)$. The lemma follows immediately.

Lemma 2.3. $\bar{X}_{g e o}(\bar{v})=c(v) d \psi \circ X_{g e o}(v)$ for all $v \in S_{g, h}$. 
Proof. Define $I: T M \rightarrow T M$ by $I(u)=-u$. Then

$$
d I\left(i_{u}\left(u^{\prime}, u^{\prime \prime}\right)\right)=i_{-u}\left(u^{\prime},-u^{\prime \prime}\right)
$$

for any vectors $u, u^{\prime}, u^{\prime \prime}$ in the same fibre of $T M$. Using this property and the linearity of the Lorenz force $Y_{\pi(v)}$, we obtain

$$
\begin{aligned}
X_{\text {mag }}(v)-d I \circ X_{\text {mag }}(-v) & =i_{v}\left(v, Y_{\pi(v)} v\right)-d I \circ i_{-v}\left(-v, Y_{\pi(-v)}(-v)\right) \\
& =i_{v}\left(v, Y_{\pi(v)} v\right)-d I \circ i_{-v}\left(-v, Y_{\pi(v)}(-v)\right) \\
& =i_{v}\left(v, Y_{\pi(v)} v\right)-i_{v}\left(-v, Y_{\pi(v)}(v)\right) \\
& =2 X_{g e o}(v) .
\end{aligned}
$$

Similarly

$$
2 \bar{X}_{\text {geo }}(\bar{v})=\bar{X}_{\text {mag }}(\bar{v})-d I\left(\bar{X}_{\text {mag }}(-\bar{v})\right) .
$$

It is obvious from the definitions that $c(v)=c(-v)$ and hence $I$ commutes with $\psi$, which entails $d I \circ d \psi=d \psi \circ d I$. Using the previous lemma and these observations, we obtain

$$
\begin{aligned}
2 \bar{X}_{\text {geo }}(\bar{v}) & =\bar{X}_{\text {mag }}(\bar{v})-d I\left(\bar{X}_{\text {mag }}(-\bar{v})\right) \\
& =c(v) d \psi \circ X_{\text {mag }}(v)-d I\left(c(-v) d \psi \circ X_{\text {mag }}(-v)\right) \\
& =c(v)\left[d \psi \circ X_{\text {mag }}(v)-d I \circ d \psi \circ X_{\text {mag }}(-v)\right] \\
& =c(v) d \psi\left[X_{\text {mag }}(v)-d I \circ X_{\text {mag }}(-v)\right] \\
& =c(v) d \psi \circ X_{\text {geo }}(v) .
\end{aligned}
$$

The proof of the lemma is complete.

It follows immediately from the previous lemma that the metrics $g$ and $\bar{g}$ are geodesically equivalent, i.e. they have the same geodesics up to reparametrization. Now let us show that the magnetic forms $\Omega$ and $\bar{\Omega}$ must vanish unless $g$ and $\bar{g}$ are homothetic.

Lemma 2.4. For every $v \in T M, v \neq 0$, we have

$$
\bar{Y}_{\pi(v)}(v)=c(v)\left[Y_{\pi(v)}(v)-\frac{\bar{g}\left(Y_{\pi(v)}(v), v\right)}{\bar{g}(v, v)} v\right] .
$$

The proof will use a simple calculation, which the reader can easily verify.

Lemma 2.5. Let $f: \mathbf{R}^{n} \rightarrow \mathbf{R}^{n}$ be given by $f(x)=\alpha(x) x$, where $\alpha: \mathbf{R}^{n} \rightarrow \mathbf{R}$ is differentiable. Then

$$
D_{x} f(w)=\alpha(x) w+\left(D_{x} \alpha(w)\right) x .
$$

Proof of Lemma 2.4. By Lemma 2.2

$$
\bar{X}_{\text {mag }}(\bar{v})=c(v) d \psi \circ X_{\text {mag }}(v) .
$$

Let $p=\pi(v)=\pi(\bar{v})$. Apply the connector map $\bar{K}$ for the the metric $\bar{g}$ to obtain

$$
\begin{aligned}
\bar{Y}_{p}(\bar{v}) & =c(v) \bar{K} \circ d \psi \circ X_{\text {mag }}(v), \\
\bar{Y}_{p}(c(v) v) & =c(v) \bar{K} \circ d \psi \circ X_{m a g}(v), \\
\bar{Y}_{p}(v) & =\bar{K} \circ d \psi \circ X_{m a g}(v) .
\end{aligned}
$$

Now

$$
X_{\text {mag }}(v)=i_{v}\left(v, Y_{p}(v)\right)=i_{v}(v, 0)+i_{v}\left(0, Y_{p}(v)\right)=X_{g e o}(v)+i_{v}\left(0, Y_{p}(v)\right) .
$$


Thus

$$
\bar{Y}_{p}(v)=\bar{K} \circ d \psi \circ X_{g e o}(v)+\bar{K} \circ d \psi \circ i_{v}\left(0, Y_{p}(v)\right) .
$$

Lemma 2.3 says that $d \psi \circ X_{g e o}(v)$ is a multiple of $\bar{X}_{g e o}(\bar{v})$, which is horizontal with respect to $\bar{g}$, i.e. lies in the kernel of $\bar{K}$. Hence

$$
\bar{Y}_{p}(v)=\bar{K} \circ d \psi \circ i_{v}\left(0, Y_{p}(v)\right) .
$$

Since $i_{v}\left(0, Y_{p}(v)\right)$ is tangent to the fiber $T_{p} M$ of $T M$ which is mapped into itself by $\psi$, the vector $d \psi \circ i_{v}\left(0, Y_{p}(v)\right)$ is tangent to $T_{p} M$; in other words $d \psi \circ i_{v}\left(0, Y_{p}(v)\right)$ is a vertical vector. Since the action of the connector map on vertical vectors is the same for all Riemannian metrics, we obtain

$$
\bar{Y}_{p}(v)=K \circ d \psi \circ i_{v}\left(0, Y_{p}(v)\right) \text {. }
$$

By applying Lemma 2.5 to the restriction of $\psi$ to $T_{p} M$, we see that $d \psi$ 。 $i_{v}\left(0, Y_{p}(v)\right)$ is equal to $i_{v}\left(0, c(v) Y_{p}(v)\right)$ plus a multiple of $i_{v}(0, v)$. Hence $\bar{Y}_{p}(v)$ is equal to $c(v) Y_{p}(v)$ plus a multiple of $v$. On the other hand we know that $\bar{Y}_{p}(v)$ is orthogonal to $v$ in the metric $\bar{g}$. Elementary Euclidean geometry in $T_{p} M$ with the inner product defined by $\bar{g}$ gives us

$$
\bar{Y}_{p}(v)=c(v) Y_{p}(v)-\frac{\bar{g}\left(c(v) Y_{p}(v), v\right)}{\bar{g}(v, v)} v .
$$

Thus equation (2) holds for every $v \in S_{g, h}$. Since both sides of the equation are homogeneous in $v$ of degree 1 , equation (2) holds for all $v \in T M$ such that $v \neq 0$. Lemma 2.4 is proved.

Lemma 2.6. $\bar{g}$ and $g$ are conformal at a point $p \in M$ unless $\Omega(p)=0=\bar{\Omega}(p)$.

Proof. It follows from Lemma 2.4 and the definition of $c(v)$ that for every $v \in T_{p} M$ such that $v \neq 0$ and $\bar{Y}_{p}(v) \neq 0$, we have

$$
\frac{g(v, v)}{\bar{g}(v, v)}=\frac{h}{\bar{h}}\left[\frac{g\left(\bar{Y}_{p}(v), \bar{Y}_{p}(v)\right)}{g\left(\overline{\bar{Y}}_{p}(v), \bar{Y}_{p}(v)\right)}\right]^{2},
$$

where

$$
\overline{\bar{Y}}_{p}(v)=Y_{p}(v)-\frac{\bar{g}\left(Y_{p}(v), v\right)}{\bar{g}(v, v)} v .
$$

The right-hand side of equation (4) is the square of a rational function of $v$. Since $\bar{g}(v, v)$ and $g(v, v)$ are irreducible polynomials in $v$, this is possible only if $\bar{g}(v, v)$ is a constant multiple of $g(v, v)$, or the numerator and denominator on the right vanish identically. In the latter case we have $\Omega(p)=0=\bar{\Omega}(p)$. In the former case $g$ and $\bar{g}$ are conformal at $p$. The lemma is proved.

We now see that, unless $\Omega(p)=0=\bar{\Omega}(p)$ for all $p$, there will be a nonempty open set on which the geodesically equivalent metrics $g$ and $\bar{g}$ are conformally equivalent. Hence, by [18, 19] (or, alternatively, see Corollary 1 from [11]), the restrictions of $g$ and $\bar{g}$ to this set are proportional (one is a constant multiple of the other). Then, since $g$ and $\bar{g}$ are geodesically equivalent by Lemma 2.3. the metrics are proportional on the whole manifold. Indeed, in view of [17] (see Corollary 2 there), if two geodesically equivalent metrics on a connected manifold of dimension at least two are proportional on an open nonempty set, the number of eigenvalues of one metric with respect to the other equals one at every point. Therefore, the 
metrics are conformally equivalent on the whole manifold. By [18, 19], the conformal coefficient is constant, so that the metrics are proportional.

Since $g$ and $\bar{g}$ are proportional on the whole manifold, $c(v)$ does not depend on $v \in S_{g, h}$. Futhermore, since $Y_{p}(v)$ and $v$ are orthogonal with respect to the metric $g$, we now have $\bar{g}\left(c(v) Y_{p}(v), v\right)=0$, and equation (3) reads

$$
\bar{Y}_{p}(v)=\operatorname{const} Y_{p}(v) \text {. }
$$

Since this is true for all $v \in S_{g, h}$, we obtain that $\Omega$ and $\bar{\Omega}$ are proportional. Finally, the magnetic system $(\bar{g}, \bar{\Omega})$ is a rescaling of $(g, \Omega)$. Theorem 1.1 is proved.

2.2. Reduction of Theorem $\mathbf{1 . 2}$ to Theorem 1.1. Suppose that there is a system $(\bar{g}, \bar{\Omega})$ whose magnetic geodesics coincide up to reparametrization with those of $(g, \Omega)$. Let $\gamma$ be a magnetic geodesic from $(g, \Omega)$ whose tangent vectors are dense in the energy level $S_{g, h}$ and let $\bar{\gamma}$ be the magnetic geodesic of $(\bar{g}, \bar{\Omega})$ that is a reparametrization of $\gamma$. Assume $\bar{\gamma}$ has energy $\bar{h}$.

The map $\psi$ defined above takes the energy level $S_{g, h}$ to the energy level $S_{\bar{g}, \bar{h}}$. Furthermore, at each tangent vector to $\gamma$, the derivative $d \psi$ maps the vector field $X_{m a g}$ generating the magnetic flow for $(g, \Omega)$ to the generator $\bar{X}_{m a g}$ of the magnetic flow for $(\bar{g}, \bar{\Omega})$. Since the vector fields $X_{m a g}$ and $\bar{X}_{m a g}$ are smooth and the tangent vectors to $\gamma$ are dense in $S_{g, h}$, it follows that $d \psi$ maps $X_{m a g}$ to $\bar{X}_{m a g}$ at all points of $S_{g, h}$. Hence the hypothesis of Theorem 1.1 holds.

\section{REFERENCES}

[1] E. Beltrami, Resoluzione del problema: riportari i punti di una superficie sopra un piano in modo che le linee geodetische vengano rappresentante da linee rette, Ann. Mat. 1 (1865), no. 7.

[2] K. Burns, G. P. Paternain, Anosov magnetic flows, critical values and topological entropy, Nonlinearity 15 (2002), no. 2, 281-314. MR1888853 (2004d:37076)

[3] N. Gouda, Magnetic flows of Anosov type, Tôhoku Math. J. 49 (1997), 165-183. MR.1447180 (98e:58129)

[4] S. Grognet, Flots magnétiques en courbure négative, Ergod. Th. and Dynam. Syst. 19 (1999), 413-436. MR1685401 (2000d:37032)

[5] S. Grognet, Entropies de flots magnétiques, Ann. Inst. H. Poincaré Phys. Théor. 71 (1999), 395-424. MR 1721559 (2000k:37035)

[6] A. Lichnerowicz, Sur la transformation des équations de la dynamique, C. R. Acad. Sci. Paris 223 (1946), 649-651. MR0018491 (8:293h)

[7] A. Lichnerowicz, D. Aufenkamp, The general problem of the transformation of the equations of dynamics, J. Rational Mech. Anal. 1 (1952), 499-520. MR0051051 (14:421a)

[8] L. Macarini, Entropy rigidity and harmonic fields, Nonlinearity 13 (2000), 1761-1774. MR:1781817 (2001h:37061)

[9] V. S. Matveev, P. J. Topalov, Trajectory equivalence and corresponding integrals, Regular and Chaotic Dynamics 3 (1998), no. 2, 30-45. MR1693470 (2000d:37068)

[10] Vladimir S. Matveev, Peter J. Topalov, Metric with ergodic geodesic flow is completely determined by unparametrized geodesics, AMS Elect. Res. Announcements 6 (2000), 98-104. MR.1796527 (2001i:37043)

[11] Vladimir S. Matveev, Hyperbolic manifolds are geodesically rigid, Invent. Math. 151 (2003), 579-609. MR1961339(2004f:53044)

[12] G. P. Paternain, M. Paternain, Anosov geodesic flows and twisted symplectic structures, International Conference on Dynamical Systems in Montevideo (a tribute to Ricardo Mañé), F. Ledrappier, J. Lewowicz, S. Newhouse, eds., Pitman Research Notes in Math. 362 (1996), 132-145. MR1460801 (98h:58145)

[13] G. P. Paternain, On the regularity of the Anosov splitting for twisted geodesic flows, Math. Res. Lett. 4 (1997), 871-888. MR1492126 (98h:58137) 
[14] G. P. Paternain, M. Paternain, First derivative of topological entropy for Anosov geodesic flows in the presence of magnetic fields, Nonlinearity 10 (1997), 121-131. MR.1430743 (97j:58118)

[15] G. P. Paternain, On two noteworthy deformations of negatively curved Riemannian metrics, Discrete Contin. Dynam. Systems. 5 (1999), 639-650. MR.1696335 (2000g:37032)

[16] N. Peyerimhoff, K. F. Siburg, The dynamics of magnetic flows for energies above Mañés critical value, Israel J. Math. 135 (2003), 269-298. MR.1997047(2004e:37098)

[17] P. J. Topalov and V. S. Matveev, Geodesic equivalence via integrability, Geometriae Dedicata 96 (2003), 91-115. MR.1956835 (2003k:53043)

[18] H. Weyl, Geometrie und Physik, Die Naturwissenschaftler 19 (1931), 49-58. Can be found in "Hermann Weyl Gesammelte Abhandlungen", Band 3, Springer Verlag, 1968.

[19] H. Weyl, Zur Infinitisimalgeometrie: Einordnung der projektiven und konformen Auffassung, Nachrichten von der K. Gesellschaft der Wissenschaften zu Göttingen, MathematischPhysikalische Klasse, 99-112, 1921. Can be found in "Selecta Hermann Weyl", Birkhaüser Verlag, 1956.

[20] M. P. Wojtkowski, Convexly hyperbolic flows on unit tangent bundles of surfaces, Tr. Mat. Inst. Steklova 216 (1997), 373-383. MR.1632202 (99i:58123)

[21] M. P. Wojtkowski, Magnetic flows and Gaussian thermostats on manifolds of negative curvature, Fund. Math. 163 (2000), no. 2, 177-191. MR1752103 (2001b:37038)

Department of Mathematics, Northwestern University, Evanston, Illinois 60208

E-mail address: burns@math.northwestern.edu

Mathematisches Institut, Universität Freiburg, 79104 Germany

E-mail address: matveev@email.mathematik.uni-freiburg.de 